\title{
On the Induced Gravitational Collapse
}

\author{
Laura M. Becerra ${ }^{1,2, \star}$, Carlo Bianco ${ }^{1,2}$, Chris Fryer ${ }^{4}$, Jorge Rueda ${ }^{1,2,3}$, and Remo Ruffini ${ }^{1,2,3}$ \\ ${ }^{1}$ Dipartimento di Fisica and ICRA, Sapienza Università di Roma, P.le Aldo Moro 5, I-00185 Rome, Italy \\ ${ }^{2}$ ICRANet, P.zza della Repubblica 10, I-65122 Pescara, Italy \\ ${ }^{3}$ ICRANet-Rio, Centro Brasileiro de Pesquisas Físicas, Rua Dr. Xavier Sigaud 150, 22290-180 Rio de \\ Janeiro, Brazil \\ ${ }^{4}$ CCS-2, Los Alamos National Laboratory, Los Alamos, NM 87545
}

\begin{abstract}
The induced gravitational collapse (IGC) paradigm has been applied to explain the long gamma ray burst (GRB) associated with type Ic supernova, and recently the $\mathrm{X}$ ray flashes (XRFs). The progenitor is a binary systems of a carbon-oxygen core $(\mathrm{CO})$ and a neutron star (NS). The CO core collapses and undergoes a supernova explosion which triggers the hypercritical accretion onto the NS companion (up to $10^{-2} M_{\odot} s^{-1}$ ). For the binary driven hypernova (BdHNe), the binary system is enough bound, the NS reach its critical mass, and collapse to a black hole (BH) with a GRB emission characterized by an isotropic energy $E_{i s o}>10^{52} \mathrm{erg}$. Otherwise, for binary systems with larger binary separations, the hypercritical accretion onto the NS is not sufficient to induced its gravitational collapse, a X-ray flash is produced with $E_{\text {iso }}<10^{52} \mathrm{erg}$. We're going to focus in identify the binary parameters that limits the BdHNe systems with the XRFs systems.
\end{abstract}

\section{Introduction}

In [1], the progenitors of long gamma-ray bursts (GRBs) have been explained within the Induced Gravitational Collapse (IGC) paradigm. The initial configuration is a compact binary system, formed by a Carbon-Oxygen (CO) core, a star that has lost its helium and hydrogen layers, with a neutron star (NS). The CO-core collapses and produces a supernova explosion that triggers an hypercritical accretion onto the NS companion. If the hypercritical accretion on the NS is not enough to make it to reach the critical mass for gravitational collapse, a first scenario takes place characterized by a X-ray flash (XRF) emission. The final system will be a NS-NS binary, formed by the $v$-NS, the remnant from the collapse of the CO-core, and a more massive NS. This kind of events are characterized by isotropic energies of $E_{\text {iso }} \lesssim 10^{52} \mathrm{erg}$, peak energies between $4<E_{\mathrm{p}, \mathrm{i}}<200 \mathrm{keV}$ and a prompt emission duration of about $\sim 10^{2}-10^{4} \mathrm{~s}$. Otherwise if the system is enough compact, the NS companion reaches by accretion the critical mass and collapses to a black hole (BH). These systems have been called binarydriven hypernovae $(\mathrm{BdHNe})$ and has been deeply studied in $[1-5,10,11]$. The BdHNe events are characterized by isotropic energies $E_{\mathrm{iso}} \gtrsim 10^{52} \mathrm{erg}$, spectral peak energy in $0.2<E_{\mathrm{p}, \mathrm{i}}<2 \mathrm{MeV}$ and prompt emission with durations of up to $100 \mathrm{~s}$. The final system is a $v \mathrm{NS}-\mathrm{BH}$ binary [6].

In this proceedings, we will identify the principal parameters of the initial binary configurations that can leave either to XRFs or BdHNe. In section 2, we estimate the accretion rate on the NS and

\footnotetext{
^e-mail: laura.maarcela@icranet.com
} 
follow its mass and angular momentum evolution in order to establish critical binary initial parameters that determined the final fate of the system after the supernova explosion. In section 3 , we model the light-curve of the XRF 060218 with the IGC scenario and finally in section 4 we resume our results.

\section{Hypercritical Accretion process}

Following the Bondy-Hoyle-Lyttleton formalism [7-9], an estimation of the accretion rate of the ejected material onto the NS is given by:

$$
\dot{M}_{B}=\pi \rho_{\mathrm{ej}} R_{\mathrm{cap}}^{2} \sqrt{v_{\mathrm{ej}}^{2}+v_{\mathrm{orb}}^{2}+c_{\mathrm{s}, \mathrm{ej}}^{2}}, \quad \text { with } \quad R_{\mathrm{cap}}=\frac{2 G M_{\mathrm{NS}}}{v_{\mathrm{ej}}^{2}+v_{\mathrm{orb}}^{2}+c_{\mathrm{s}, \mathrm{ej}}^{2}}
$$

where $R_{\text {cap }}$ is the NS gravitational capture radius, $G$ is the gravitational constant, $\rho_{\text {ej }}$ and $\vec{v}_{\text {ej }}$ the density and velocity of the supernova ejecta, $M_{\mathrm{NS}}$ the NS mass, $v_{\mathrm{orb}}=\sqrt{G\left(M_{\mathrm{NS}}+M_{\mathrm{CO}}\right) / a}$ is the orbital velocity of the binary system, with $a$ the binary separation, and $M_{\mathrm{CO}}=M_{\mathrm{env}}+M_{\mathrm{Fe}}$ the total mass of the $\mathrm{CO}$ core which consist of the envelope mass $M_{\text {env }}$ that will be ejected in the SN explosion and the central iron core mass, $M_{\mathrm{Fe}}$. In order to integrate equation (1), we have fitted the pre-supernova density profile of the $\mathrm{CO}$ envelope with a power-law, $\rho_{\mathrm{ej}} \propto r^{-m}$ with $m<3.0$, and adopted an homologous explosion model for the supernova expansion (see $[10,11]$ for details ). The left panel of figure 1 shows the evolution of the mass accretion rate onto the NS. We have used, as our canonical model, an initial binary system of a $2 M_{\odot}$ NS and the CO-core of a $30 M_{\text {zams }}$ progenitor, that ejects $M_{\text {env }}=$ $7.94 M_{\odot}$ in the SN explosion and leaves an iron core of $M_{\mathrm{Fe}}=1.5 M_{\odot}$. The accretion on the NS can be as high as $\sim 10^{-1} M_{\odot} s^{-1}$. For these high accretions, the photons would be trapped in the accretion flow and the gain of gravitational energy of the accreted material is mainly radiated via neutrino emission, specifically, by the $e^{+} e^{-}$pair annihilation process [12-14].

\subsection{Mass and angular momentum evolution of the NS companion during the hypercritical accretion}

If we want to discriminate the binary parameters of the systems in which the NS can reach, by accretion, its critical mass $\left(M_{\text {crit }}\right)$ and consequently collapse to a $\mathrm{BH}$, from the systems in which the

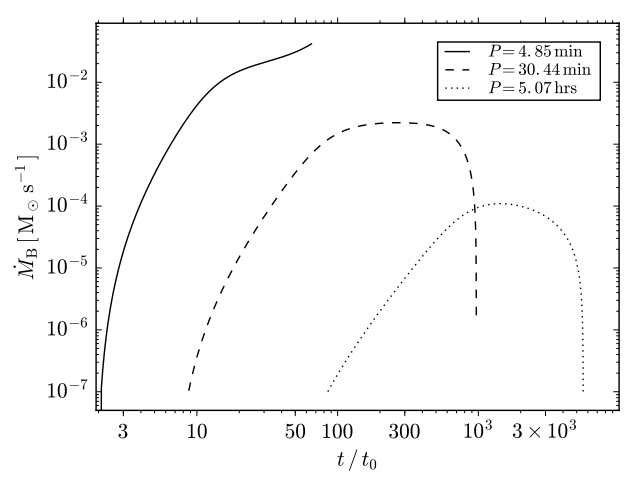

(a)

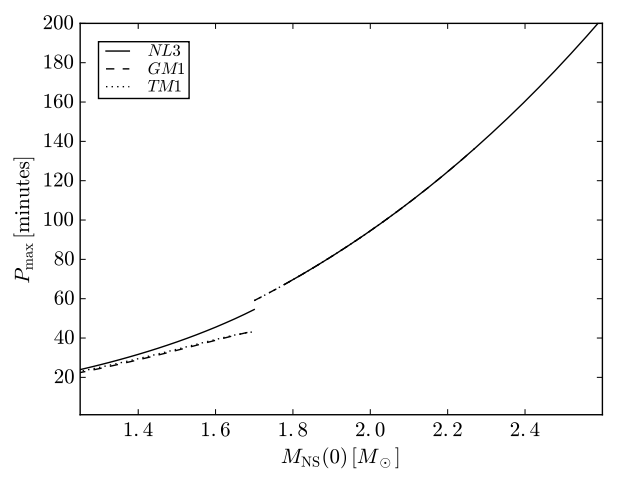

(b)

Figure 1. Left: Evolution of the mass accretion rate on the NS companion calculated with the Bondy-HoyleLyttleton accretion formalism for three selected orbital period. The initial binary system is formed by a $2 M_{\odot}$ NS and the CO-core of a $30 M_{\text {zamns }}$ progenitor. Right: Maximum orbital period for which the NS reaches its critical mass by the accretion of material from the $\mathrm{SN}$ ejecta and collapse in a $\mathrm{BH}$. 


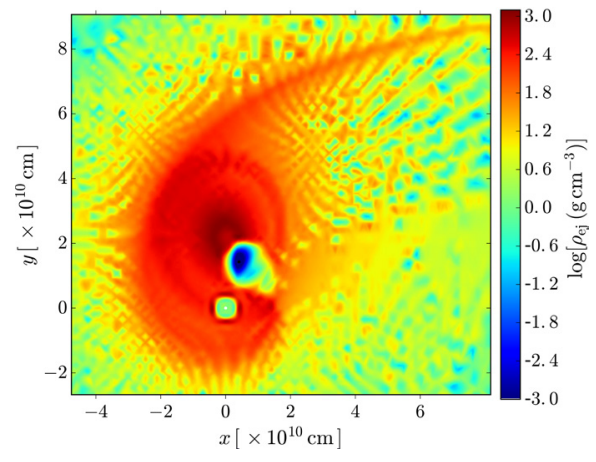

(a)

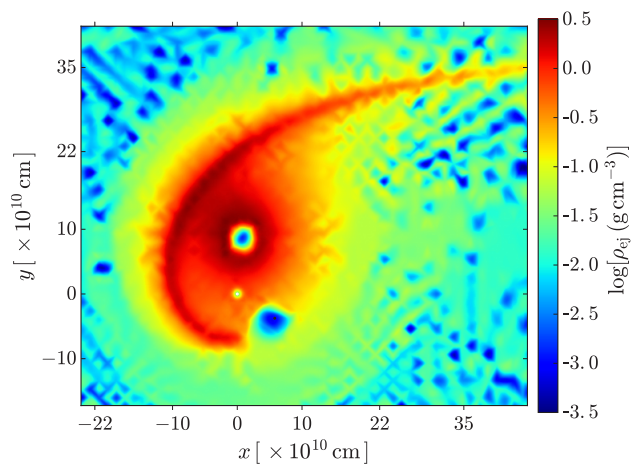

(b)

Figure 2. Snapshots of the supernova ejecta density on the binary equatorial plane in the IGC scenario. We used the binary parameter of our canonical model. The left panel corresponds to a binary initial period of $P_{\text {orb }}=5 \mathrm{~min}$ and the picture is made at $100 \mathrm{~s}$ after the collapse of the NS. The right panel corresponds to a period of $P_{\text {orb }}=50 \mathrm{~min}$. In this case the NS does not collapse and the snapshot is at $t \approx 44 \mathrm{~min}$.

accretion is not sufficient to induce such a collapse, we need to determine how the NS evolves during the accretion process. In general, the accretion of baryonic mass, $M_{b}$, as well as the accretion of angular momentum $J_{\mathrm{NS}}$, will modify the NS gravitational mass, $M_{\mathrm{NS}}$ in the following way:

$$
\dot{M}_{\mathrm{NS}}(t)=\frac{\partial M_{\mathrm{NS}}}{\partial M_{b}} \dot{M}_{b}+\frac{\partial M_{\mathrm{NS}}}{\partial J_{\mathrm{NS}}} \dot{J}_{\mathrm{NS}}
$$

In [10] it was demonstrated that the supernova ejecta material has enough angular momentum to circularize around the NS and form a disk-like structure. Then, the NS angular momentum evolution is dictated by the disk accretion torque: $\dot{J}_{\mathrm{NS}}=\xi l\left(R_{\mathrm{in}}\right) \dot{M}_{B}$, with $l\left(R_{\text {in }}\right)$ the angular momentum per unit mass of the material located at $r=R_{\text {in }}$, the disk inner boundary radius, that will be the maximum between the radius of the last stable circular orbit, $r_{\text {Iso }}$, and the NS radius, $R_{\mathrm{NS}}$. We have also introduced a parameter $\xi \leq 1$ that accounts for the efficiency of the angular momentum transfer. Additionally, if we assume that all the mass entering the NS capture region will be accreted by the NS as baryonic mass: $M_{b}(t)=M_{b}\left(t_{0}\right)+M_{B}(t)$, then $\dot{M}_{b}=\dot{M}_{B}$, we can integrate equation (2) to follow the evolution of the NS during the accretion process. We have used the fitting formulas obtained in $[15,16]$ for the relations between the NS gravitational mass with its baryonic mass and angular momentum as well as the angular momentum of the last stable orbit.

Thus, a NS with initial mass $M_{\mathrm{NS}}\left(t_{0}\right)$ can reach $M_{\text {crit }}$ if it accretes an amount of mass $\Delta M_{\text {acc }}=$ $M_{\text {crit }}-M_{\mathrm{NS}}\left(t_{0}\right)$ from the supernova ejecta. This critical mass will depend, in general, on the EoS consider to model the NS matter and, in the case of a rotating NS, $\mathrm{n}$ the star angular momentum [15]. Therefore, given the initial NS mass, the CO core mass, and the supernova ejecta profile and its velocity, the accretion rate increases for shorter orbital periods. Therefore, there exists a maximum orbital period, $P_{\max }$, up to which, given $M_{\mathrm{NS}}\left(t_{0}\right)$ (and all the other binary parameters), the NS can accreate this precise amount of mass, $\Delta M_{\text {acc }}$. The right panel of figure 1 shows $P_{\max }$ as a function of the NS initial mass, for a $\mathrm{CO}$ core progenitor of $30 M_{\mathrm{zmans}}$.

\section{$3 \mathrm{SN}$-asymmetries induced by the NS companion presence}

For supernova explosions occurring in close binaries with compact companions such as NSs or BHs, like the case of the IGC scenario, the supernova ejecta is subjected to a strong gravitational field 


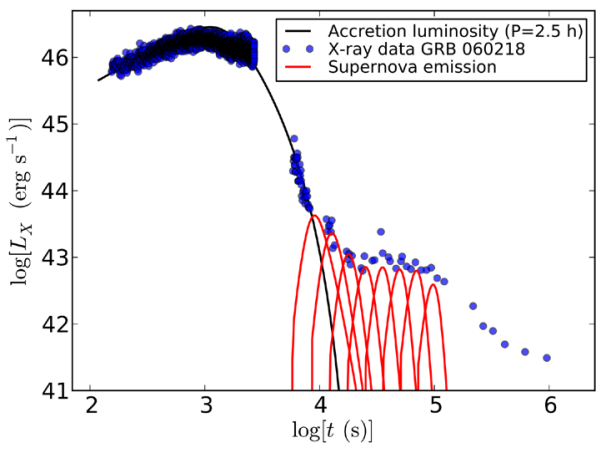

(a)

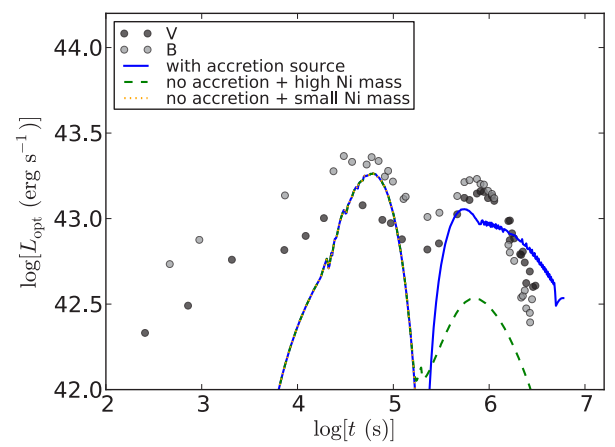

(b)

Figure 3. Left: Comparison of the accretion and the supernova luminosity with the observed X-ray luminosity of XRF 060218. The binary system has the following parameters: supernova velocity $v_{\text {star }, 0}=2 \times 10^{9} \mathrm{~cm} \mathrm{~s}^{-1}$, a pre-supernova core obtained from the $M_{\mathrm{ZAMS}}=20 M_{\odot}$ evolution, initial NS mass $M_{\mathrm{NS}}\left(t_{0}\right)=1.4 M_{\odot}$, and orbital period of $2.5 \mathrm{~h}$. Right: Optical and UV luminosity of XRF 060218 [18]. The red dotted curve shows the supernova optical emission without either ${ }^{56} \mathrm{Ni}$ decay or accretion energy. The blue solid curve includes the energy deposition from the accretion onto the NS. The dashed green curve increases the total ${ }^{56} \mathrm{Ni}$ yield but cannot explain the observational data.

which produces a deformation of the supernova fronts closer to the accreting companion. In order to visualize this, we have simulated the evolution of the supernova layers in the binary system by dividing the SN ejecta in $N$ particles of different mass and following its three-dimensional motion under the action of the gravitational field of the orbiting NS. We have varied the NS gravitational mass with equations (1) and (2) and also, we have removed from the simulation the particles that are crossing the Bondi-Hoyle radius. Figure 2 shows the surface density on the equatorial plane for the canonical initial binary system parameters. The left panel corresponds to an orbital period of about $P \approx 5$ min and its made at $100 \mathrm{~s}$ after the collapse of the NS companion and the formation of the $\mathrm{BH}$. It can be seen the increasing asymmetry of the supernova ejecta around the orbital plane. Even for longer binary periods, from which the accretion does not lead to the collapse of the NS, the asymmetries can still be formed, as in shown in the right panel of figure 2 . This system corresponds to a orbital period of about $P \approx 50 \mathrm{~min}$.

In order to validate the IGC scenario, we have to contrast it with the observations. In the left panel of figure 3 we present the observed X-ray luminosity of XRF 060218. The early part of the lightcurve $\left(t \lesssim 10^{3} \mathrm{~s}\right)$ has been fitted with the luminosity expected from the accretion process on the NS companion, that was estimated as: $L_{\mathrm{acc}}=\left(\dot{M}_{b}-\dot{M}_{\mathrm{NS}}\right) c^{2}$, the amount of gravitational energy gained by the accreted matter by falling to the NS surface and which is not spent in changing the gravitational binding energy of the NS.

For the long-lasting X-ray plateau in the afterglow (at times $t \sim 10^{3}-10^{6} \mathrm{~s}$ ) we need to analyze the emission of the supernova at early stages. We have calculated the shock breakout luminosity using the light-curve code described in [17]. To simulate the energy that the hypercritical accretion process onto the NS adds to the ejecta, we injected it as an energy source at the base of the explosion and to mimic the asymmetries in the SN ejecta, caused by the NS companion presence (see figures 2), we have modeled a series of spherical explosions with different densities. For XRF 060218, we assume an initial explosion energy of $2 \times 10^{51} \mathrm{erg}$, ranging the spherical equivalent-mass from $0.05-4 M_{\odot}$. Figure 3 shows light-curves rising quickly at $t \lesssim 10^{4} \mathrm{~s}$ for the lowest mass to $\sim 10^{5} \mathrm{~s}$ for the $4 M_{\odot}$ 
explosion. The observed emission would come from the sum of this full range of explosions (see [11] for details).

Finally, the right panel of figure 3 shows the V and B band light-curves for XRF 060218 [18]. The light-curve has two peaks: near 50,000 s and at 500,000 s. Using our $1 M_{\odot} 1 \mathrm{D}$ model from our X-ray emission, we simulate the $\mathrm{V}$ and $\mathrm{B}$ band light-curves. Without either ${ }^{56} \mathrm{Ni}$ decay or accretion energy, the supernova explosion only explains the first peak. However, if we include the energy deposition from the accretion onto the NS (for our energy deposition, we use $4 \times 10^{46} \mathrm{erg} \mathrm{s}^{-1}$ over a $2500 \mathrm{~s}$ duration), our simulations produce a second peak at roughly 500,000 s (see [11] for details).

\section{Conclusions}

We have studied the IGC paradigm to explain the nature of BdHNe and XRFs. We have followed the accretion onto the NS and computed the binary period, $P_{\max }$, over which the NS does not accrete sufficient matter to reach the critical mass and produce a $\mathrm{BH}$. We have shown that the presence of the NS in very compact orbit produces large asymmetries in the supernova ejecta around the orbital plane due to the accretion and the action of the NS gravitational field on the supernova layers. These asymmetries lead to observable effects in the supernova emission. We have analyzed the light curve of XRF 060218 and explained its prompt emission $\left(t \lesssim 10^{3} \mathrm{~s}\right)$ with the accretion luminosity. We have shown that the observed long-lasting $\left(t>10^{3} \mathrm{~s}\right)$ afterglow X-ray emission can be powered by a sequence of shock breakouts in different directions: the more massive directions produce later shock breakouts. Finally, we have simulated the optical emission of the supernova and compared our theoretical expectation with the optical luminosity of XRF 060218 which shows a peculiar doublepeaked shape. We demonstrated that the source of energy given by the hypercritical accretion onto the NS provides a double-peaked light-curve consistent with the observational data.

\section{References}

[1] Ruffini, R., Rueda, J. A., Muccino, M., et al., ApJ 832, 136 (2016)

[2] Ruffini, R., Bernardini, M. G., Bianco, C. L., et al. Proceedings of the MG10 Meeting (Singapore: World Scientific Publishing, Rio de Janeiro, Brazil, 2003) 369

[3] Izzo, L., Rueda, J. A., \& Ruffini, R., A\&A 548, L5 (2012)

[4] Rueda, J. A., \& Ruffini, R., ApJL, 758, L7 (2012)

[5] Fryer, C. L. and Rueda, J. A. and Ruffini, R., ApJL, 793, L36 (2014)

[6] Fryer, C. L. and Oliveira, F. G. and Rueda, J. A. and Ruffini, R., PhRvL, 115, 231102 (2015)

[7] Hoyle, F., \& Lyttleton, R. A., Proceedings of the Cambridge Philosophical Society 35, 405 (1939)

[8] Bondi, H., \& Hoyle, F., MNRAS 104, 273 (1944)

[9] Bondi, H., MNRAS 112, 195 (1952)

[10] Becerra, L., Cipolletta, F., Fryer, C. L., Rueda, J. A., \& Ruffini, R., ApJ 812, 100 (2015)

[11] Becerra, L., Bianco, C., Fryer, C. L., Rueda, J. A., \& Ruffini, R., ApJ 833, 107 (2016)

[12] Zel'dovich, Y. B., Ivanova, L. N., \& Nadezhin, D. K., Soviet Ast 16, 209 (1972)

[13] Chevalier, R. A., ApJ 346, 847 (1989)

[14] Fryer, C. L., Benz, W., \& Herant, M., ApJ 460, 801 (1996)

[15] Cipolletta, F., Cherubini, C., Filippi, S., Rueda, J. A., \& Ruffini, R., PhRvD 92, 023007 (2015)

[16] Cipolletta, F. and Cherubini, C. and Filippi, S. and Rueda, J. A., \& Ruffini, R., PhRvD 96, 024046 (2017)

[17] Bayless, A. J., Even, W., Frey, L. H., et al., ApJ 805, 98 (2015)

[18] Pian, E., Mazzali, P. A., Masetti, N., et al. 2006, Nature 442, 1011 (2006) 